\title{
Asymptotic performance analysis of reception diversity with coherent detection in Nakagami-Rice fading channels
}

\author{
Masaaki Harada ${ }^{a}$ \\ a)m.harada@m.ieice.org
}

\begin{abstract}
This paper analyzes the average bit error rate performance of reception diversity in fading channels. The analysis provides the upper bounds on the bit error rate probability for differentially coherent detection in correlated Nakagami-Rice fading channels and coherent detection with channel phase information in uncorrelated Nakagami-Rice fading channels. Both bounds are derived by developing the Chernoff bound for the pairwise error probabilities. Numerical examples compare the analysis bounds with the simulation results.
\end{abstract}

Keywords: reception diversity, differential detection, equal gain combining, correlated fading channel

Classification: Wireless Communication Technologies

\section{References}

[1] P. Y. Kam, "Bit error probabilities of MDPSK over the nonselective Rayleigh fading channel with diversity reception," IEEE Trans. Commun., vol. 39, no. 2, pp. 220-224, Feb. 1991.

[2] F. Adachi and M. Sawahashi, "Error rate analysis of MDPSK/CPSK with diversity reception under very slow Rayleigh fading and cochannel interference," IEEE Trans. Veh. Technol., vol. 43, no. 2, pp. 252-262, May 1994.

[3] S. Zhang, P. Y. Kam, and P. Ho, "Performance of differentially detected DPSK over nonselective Rayleigh fading channels with maximal ratio combining and multiple cochannel interferers," IEEE Trans. Commun., vol. 55, no. 1, pp. 133-141, Jan. 2007.

[4] D. A. Zogas, G. K. Karagiannidis, and S. A. Kotsopoulos, "Equal gain combining over Nakagami- $n$ (Rice) and Nakagami- $q$ (Hoyt) generalized fading channels," IEEE Trans. Wireless Commun., vol. 4, no. 2, pp. 374379, March 2005.

[5] N. Sagias, "Closed-form analysis of equal-gain diversity in wireless radio networks," IEEE Trans. Veh. Technol., vol. 56, pp. 173-182, Jan. 2007.

[6] D. Divsalar and M. K. Simon, "Trellis coded modulation for 4800$9600 \mathrm{bits} / \mathrm{s}$ transmission over a fading mobile satellite channel," IEEE $J$. Sel. Areas Commun., vol. 5, no. 2, pp. 162-175, Feb. 1987.

[7] M. K. Simon and D. Divsalar, "The performance of trellis coded multilevel DPSK on a fading mobile satellite channel," IEEE Trans. Veh. Technol., vol. 37, no. 2, pp. 78-91, May 1988. 
[8] I. M. Jacobs, "Probability-of-error bounds for binary transmission on the slowly fading Rician channel," IEEE Trans. Inf. Theory, vol. 12, no. 4, pp. 431-441, Oct. 1966.

[9] A. Dogandzic, "Chernoff bounds on pairwise error probabilities of spacetime codes," IEEE Trans. Inf. Theory, vol. 49, no. 5, pp. 1327-1336, May 2003.

\section{Introduction}

Diversity reception is a promising method to reduce the receive power fluctuation in fading channels. A lot of research studies have been carried out for several combining and detection methods. Differentially coherent detection is one of the detection schemes, which recovers a phase ambiguity from a previously received symbol. Related work concerning differential detection reception diversity has been studied such as in $[1,2,3]$. The closed-form expression of the exact bit error rate (BER) probability for differential phaseshift keying in uncorrelated Rayleigh fading channels was provided in [1]. The performance for other scenarios, such as under cochannel interference, has been analyzed in $[2,3]$.

Equal gain combining is another detection and combining scheme. This method detects the signal only with channel phase information and hence has advantages such as detectability under insufficient channel state information and reduction of receiver complexity. Several papers such as [4] and [5] have studied the performance in fading channels. [4] analyzed the error probabilities for Nakagami-Rice and Nakagami- $q$ fading channels. The closed-form expression for Nakagami- $m$ fading channels was derived in [5] by using the lower bound for the sum of Nakagami- $m$ fading envelopes. Most of the studies for equal gain combining have not led to closed-form because of the difficulty of finding the probability density function of the sum of fading envelopes.

This paper analyzes the performance of reception diversity with coherent detection in Nakagami-Rice fading channels. The analysis takes another approach using the Chernoff bound of the pairwise error probability based on [6] and [7]. This type of the approach has been often used in the performance studies of error-correcting codes. Some studies on diversity systems have used the similar approach such as in [8], which showed the closed-form expression of the BER probability for square-law combining reception diversity in Nakagami-Rice fading channels. The analysis in this study derives the BER probabilities for differentially coherent detection in correlated Nakagami-Rice fading channels and coherent detection with channel phase information in uncorrelated Nakagami-Rice fading channels. The detection with phase information is also referred to as equal gain combining. Numerical examples compare the analysis bounds with the simulation results. 


\section{System model}

Consider a diversity reception system over a flat fading channel with $N_{\mathrm{R}}$ branches, in which the receiver employs symbol-by-symbol detection. The signal received at a given time instance $n_{\mathrm{t}}$ over the diversity branches can be represented as

$$
\boldsymbol{y}_{n_{\mathrm{t}}}=\boldsymbol{h} s_{n_{\mathrm{t}}}+\boldsymbol{n}_{n_{\mathrm{t}}},
$$

where $\boldsymbol{h} \in \mathbb{C}^{N_{\mathrm{R}} \times 1}$ is the channel coefficient vector, $s_{n_{\mathrm{t}}}$ is the transmit symbol, and $\boldsymbol{n}_{n_{\mathrm{t}}} \in \mathbb{C}^{N_{\mathrm{R}} \times 1}$ is the complex circularly symmetric additive white Gaussian noise (AWGN) having the relation of $E\left[\boldsymbol{n}_{n_{\mathrm{t}}} \boldsymbol{n}_{n_{\mathrm{t}}}^{\mathrm{H}}\right]=\sigma_{\mathrm{N}}^{2} \mathbf{I}_{N_{\mathrm{R}}}$, where $E$ denotes the statistical expectation, $\mathbf{I}_{n}$ is the $n \times n$ identity matrix, and $(\cdot)^{\mathrm{H}}$ denotes the Hermitian transpose. The detector calculates the symbol metrics as

$$
m\left(\boldsymbol{y}_{n_{\mathrm{t}}}, \hat{x}_{n_{\mathrm{t}}} ; \boldsymbol{w}_{n_{\mathrm{t}}}\right)=\ln p_{\mathrm{N}}\left(\boldsymbol{y}_{n_{\mathrm{t}}} ; \hat{x}_{n_{\mathrm{t}}}, \boldsymbol{w}_{n_{\mathrm{t}}}\right),
$$

where $p_{\mathrm{N}}(\cdot)$ denotes the channel probability, $\hat{x}_{n_{\mathrm{t}}}$ is the estimated symbol, and $\boldsymbol{w}_{n_{\mathrm{t}}} \in \mathbb{C}^{N_{\mathrm{R}} \times 1}$ is the weight vector at the receiver. Since the noise is AWGN, Eq. (2) is replaced with

$$
m\left(\boldsymbol{y}_{n_{\mathrm{t}}}, \hat{x}_{n_{\mathrm{t}}} ; \boldsymbol{w}_{n_{\mathrm{t}}}\right)=-\left\|\boldsymbol{y}_{n_{\mathrm{t}}}-\boldsymbol{w}_{n_{\mathrm{t}}} \hat{x}_{n_{\mathrm{t}}}\right\|^{2},
$$

where $\|\cdot\|^{2}$ denotes the squared Euclidean norm of the vector. The detector decides the symbol that shows the maximum value of the metric as the information symbol. The task is written as

$$
\hat{x}_{n_{\mathrm{t}}}=\underset{\hat{x}_{n_{\mathrm{t}}} \in \mathcal{X}}{\operatorname{argmax}}-\left\|\boldsymbol{y}_{n_{\mathrm{t}}}-\boldsymbol{w}_{n_{\mathrm{t}}} \hat{x}_{n_{\mathrm{t}}}\right\|^{2},
$$

where $\mathcal{X}$ denotes the set of all symbols.

\section{Asymptotic analysis}

On the basis of the approach in [6] and [7], the Chernoff bound is developed to express the pairwise error probabilities. The phase-shift keying signal is assumed throughout the analysis. Consider that the detector chooses the symbol $\hat{x}_{n_{\mathrm{t}}}$ incorrectly as the information symbol. The erroneous detection occurs when the metric of the incorrect symbol $\hat{x}_{n_{\mathrm{t}}}$ is larger than that of the correct symbol $x_{n_{\mathrm{t}}}$, namely,

$$
m\left(\boldsymbol{y}_{n_{\mathrm{t}}}, \hat{x}_{n_{\mathrm{t}}} ; \boldsymbol{w}_{n_{\mathrm{t}}}\right) \geq m\left(\boldsymbol{y}_{n_{\mathrm{t}}}, x_{n_{\mathrm{t}}} ; \boldsymbol{w}_{n_{\mathrm{t}}}\right) .
$$

The pairwise error probability between $x_{n_{\mathrm{t}}}$ and $\hat{x}_{n_{\mathrm{t}}}$ is written as

$$
P\left(x_{n_{\mathrm{t}}}, \hat{x}_{n_{\mathrm{t}}}\right)=P\left(m\left(\boldsymbol{y}_{n_{\mathrm{t}}}, \hat{x}_{n_{\mathrm{t}}} ; \boldsymbol{w}_{n_{\mathrm{t}}}\right) \geq m\left(\boldsymbol{y}_{n_{\mathrm{t}}}, x_{n_{\mathrm{t}}} ; \boldsymbol{w}_{n_{\mathrm{t}}}\right)\right) .
$$

Equation (6) is bounded using the Chernoff bound as

$$
P\left(x_{n_{\mathrm{t}}}, \hat{x}_{n_{\mathrm{t}}}\right) \leq E\left[\exp \left\{-\lambda\left(m\left(\boldsymbol{y}_{n_{\mathrm{t}}}, \hat{x}_{n_{\mathrm{t}}} ; \boldsymbol{w}_{n_{\mathrm{t}}}\right)-m\left(\boldsymbol{y}_{n_{\mathrm{t}}}, x_{n_{\mathrm{t}}} ; \boldsymbol{w}_{n_{\mathrm{t}}}\right)\right)\right\}\right],(7)
$$

where $\lambda$ is the parameter of the Chernoff bound to be optimized. 
For coherent detection with ideal channel information, the weight vector and transmit symbol are given by $\boldsymbol{w}_{n_{\mathrm{t}}}=\boldsymbol{h}$ and $s_{n_{\mathrm{t}}}=x_{n_{\mathrm{t}}}$, respectively. Substituting into Eq. (7) and taking the expectation over the noise rewrites the bound as

$$
P\left(x_{n_{\mathrm{t}}}, \hat{x}_{n_{\mathrm{t}}}\right) \leq \exp \left(-\lambda\left\|\boldsymbol{h}\left(x_{n_{\mathrm{t}}}-\hat{x}_{n_{\mathrm{t}}}\right)\right\|^{2}\left(1-\lambda \sigma_{\mathrm{N}}^{2}\right)\right) .
$$

Optimizing Eq. (8) over the Chernoff bound parameter $\lambda$ yields

$$
\lambda_{\mathrm{o}}=\frac{1}{2 \sigma_{\mathrm{N}}^{2}} .
$$

The channel is assumed to be distributed according to the correlated Nakagami-Rice distribution. Substituting Eq. (9) into Eq. (8) and averaging, the bound is derived as [9]

$$
P\left(x_{n_{\mathrm{t}}}, \hat{x}_{n_{\mathrm{t}}}\right) \leq \frac{\exp \left(-\frac{\left|x_{n_{\mathrm{t}}}-\hat{x}_{n_{\mathrm{t}}}\right|^{2}}{4 \sigma_{\mathrm{N}}^{2}} \boldsymbol{m}^{\mathrm{H}}\left(\mathbf{I}_{N_{\mathrm{R}}}+\frac{\left|x_{n_{\mathrm{t}}}-\hat{x}_{n_{\mathrm{t}}}\right|^{2}}{4 \sigma_{\mathrm{N}}^{2}} \boldsymbol{C}\right)^{-1} \boldsymbol{m}\right)}{\operatorname{det}\left(\mathbf{I}_{N_{\mathrm{R}}}+\frac{\left|x_{n_{\mathrm{t}}}-\hat{x}_{n_{\mathrm{t}}}\right|^{2}}{4 \sigma_{\mathrm{N}}^{2}} \boldsymbol{C}\right)},
$$

where $\boldsymbol{C}$ and $\boldsymbol{m}$ are the covariance and mean of the fading vector, respectively. $|\cdot|$ is the absolute value, and $\operatorname{det}(\cdot)$ denotes the determinant of the matrix.

If the receiver detects the signal with differentially coherent detection, the weight vector is given by the previously received signals, namely, $\boldsymbol{w}_{n_{\mathrm{t}}}=$ $\boldsymbol{y}_{n_{\mathrm{t}}-1}$, and the transmit symbol becomes $s_{n_{\mathrm{t}}}=x_{n_{\mathrm{t}}} s_{n_{\mathrm{t}}-1}$. Substituting into Eq. (7), the bound is written in the form [7]

$$
P\left(x_{n_{\mathrm{t}}}, \hat{x}_{n_{\mathrm{t}}}\right) \leq E\left[\exp \left(-\lambda \boldsymbol{v}^{\mathrm{H}} \boldsymbol{X} \boldsymbol{v}\right)\right]
$$

where

$$
\begin{aligned}
\boldsymbol{v} & =\left[\begin{array}{c}
\boldsymbol{y}_{n_{\mathrm{t}}-1} \\
\boldsymbol{y}_{n_{\mathrm{t}}}
\end{array}\right], \\
\boldsymbol{X} & =\left[\begin{array}{cc}
\mathbf{0}_{N_{\mathrm{R}}} & \left(x_{n_{\mathrm{t}}}-\hat{x}_{n_{\mathrm{t}}}\right)^{*} \mathbf{I}_{N_{\mathrm{R}}} \\
\left(x_{n_{\mathrm{t}}}-\hat{x}_{n_{\mathrm{t}}}\right) \mathbf{I}_{N_{\mathrm{R}}} & \mathbf{0}_{N_{\mathrm{R}}}
\end{array}\right] .
\end{aligned}
$$

$\mathbf{0}_{n}$ and $(\cdot)^{*}$ in Eq. (13) denote the $n \times n$ zero matrix and the complex conjugate, respectively. The expectation of Eq. (11) over the noise rewrites the bound as

$$
P\left(x_{n_{\mathrm{t}}}, \hat{x}_{n_{\mathrm{t}}}\right) \leq \frac{\exp \left(-\lambda \boldsymbol{\mu}^{\mathrm{H}} \boldsymbol{X}\left(\mathbf{I}_{2 N_{\mathrm{R}}}+\lambda \sigma_{\mathrm{N}}^{2} \boldsymbol{X}\right)^{-1} \boldsymbol{\mu}\right)}{\operatorname{det}\left(\mathbf{I}_{2 N_{\mathrm{R}}}+\lambda \sigma_{\mathrm{N}}^{2} \boldsymbol{X}\right)},
$$

where

$$
\boldsymbol{\mu}=E\left[\begin{array}{c}
\boldsymbol{y}_{n_{\mathrm{t}}-1} \\
\boldsymbol{y}_{n_{\mathrm{t}}}
\end{array}\right] .
$$

Correlated Nakagami-Rice fading is assumed as in the previous case. Averaging Eq. (14) over the channel, the bound is derived as

$$
P\left(x_{n_{\mathrm{t}}}, \hat{x}_{n_{\mathrm{t}}}\right) \leq \frac{\exp \left(-\lambda \boldsymbol{m}^{\mathrm{H}} \boldsymbol{S}^{\mathrm{H}} \boldsymbol{\Phi}\left(\mathbf{I}_{2 N_{\mathrm{R}}}+\lambda \boldsymbol{R} \boldsymbol{\Phi}\right)^{-1} \boldsymbol{S} \boldsymbol{m}\right)}{\operatorname{det}\left(\mathbf{I}_{2 N_{\mathrm{R}}}+\lambda\left(\sigma_{\mathrm{N}}^{2} \mathbf{I}_{2 N_{\mathrm{R}}}+\boldsymbol{R}\right) \boldsymbol{X}\right)},
$$


where

$$
\begin{aligned}
\boldsymbol{\Phi} & =\boldsymbol{X}\left(\mathbf{I}_{2 N_{\mathrm{R}}}+\lambda \sigma_{\mathrm{N}}^{2} \boldsymbol{X}\right)^{-1}, \\
\boldsymbol{R} & =\left[\begin{array}{cc}
1 & x_{n_{\mathrm{t}}}^{*} \\
x_{n_{\mathrm{t}}} & 1
\end{array}\right] \otimes \boldsymbol{C}, \\
\boldsymbol{S} & =\left[\begin{array}{c}
1 \\
x_{n_{\mathrm{t}}}
\end{array}\right] \otimes \mathbf{I}_{N_{\mathrm{R}}} .
\end{aligned}
$$

$\otimes$ in Eqs. (18) and (19) denotes the Kronecker product. In Rayleigh fading channels, Eq. (16) is simplified to

$$
P\left(x_{n_{\mathrm{t}}}, \hat{x}_{n_{\mathrm{t}}}\right) \leq \operatorname{det}^{-1}\left(\mathbf{I}_{2 N_{\mathrm{R}}}+\lambda\left(\sigma_{\mathrm{N}}^{2} \mathbf{I}_{2 N_{\mathrm{R}}}+\boldsymbol{R}\right) \boldsymbol{X}\right) .
$$

The optimum value of the Chernoff bound parameter is derivable when the diversity links are independent and identically distributed Rayleigh fading channels. The parameter is given by

$$
\lambda_{\mathrm{o}}=\frac{1}{2\left(2 \sigma_{\mathrm{N}}^{2}+\sigma_{\mathrm{N}}^{4}\right)} .
$$

If only the phase information of the propagation channels is available at the receiver, the detector calculates the metrics by using the phase vector as the weight vector. The transmit symbol is identical to the information symbol and given by $s_{n_{\mathrm{t}}}=x_{n_{\mathrm{t}}}$. Let $\boldsymbol{\vartheta} \in \mathbb{C}^{N_{\mathrm{R}} \times 1}$ denote the channel phase vector. Substituting into Eq. (7) and taking the expectation over the noise, the bound is rewritten as

$$
\begin{aligned}
& P\left(x_{n_{\mathrm{t}}}, \hat{x}_{n_{\mathrm{t}}}\right) \leq \exp [-2 \lambda \Re\left\{\left(x_{n_{\mathrm{t}}} \boldsymbol{h}\right)^{\mathrm{H}}\left(x_{n_{\mathrm{t}}}-\hat{x}_{n_{\mathrm{t}}}\right) \boldsymbol{\vartheta}\right\} \\
&\left.+\lambda^{2} \sigma_{\mathrm{N}}^{2}\left|x_{n_{\mathrm{t}}}-\hat{x}_{n_{\mathrm{t}}}\right|^{2}\right],
\end{aligned}
$$

where $\Re(\cdot)$ denotes the real part of the complex variable. The channel vector is rewritten by using the amplitude and phase vectors as

$$
\boldsymbol{h}=\operatorname{diag}(\boldsymbol{\rho}) \boldsymbol{\vartheta}
$$

where $\operatorname{diag}(\cdot)$ denotes the diagonal matrix formed with its vector argument and $\rho \in \mathbb{R}^{N_{\mathrm{R}} \times 1}$ is the channel amplitude vector. The bound is derivable if the channel is uncorrelated. Substituting Eq. (23) into Eq. (22) and averaging, the bound becomes [6]

$$
P\left(x_{n_{\mathrm{t}}}, \hat{x}_{n_{\mathrm{t}}}\right) \leq \prod_{n_{\mathrm{R}}=1}^{N_{\mathrm{R}}} \frac{2\left(1+\frac{\left|m_{n_{\mathrm{R}}}\right|^{2}}{c_{n_{\mathrm{R}}}}\right) \exp \left(\lambda^{2} \sigma_{\mathrm{N}}^{2}\left|x_{n_{\mathrm{t}}}-\hat{x}_{n_{\mathrm{t}}}\right|^{2}-\frac{\left|m_{n_{\mathrm{R}}}\right|^{2}}{c_{n_{\mathrm{R}}}}\right)}{\lambda^{2}\left(c_{n_{\mathrm{R}}}+\left|m_{n_{\mathrm{R}}}\right|^{2}\right)\left|x_{n_{\mathrm{t}}}-\hat{x}_{n_{\mathrm{t}}}\right|^{4}},
$$

where $c_{n_{\mathrm{R}}}$ is the $n_{\mathrm{R}}$-th diagonal entry of $\boldsymbol{C}$, and $m_{n_{\mathrm{R}}}$ is the $n_{\mathrm{R}}$-th entry of $\boldsymbol{m}$. The optimum parameter of the Chernoff bound is given by

$$
\lambda_{\mathrm{o}}^{2}=\frac{1}{\sigma_{\mathrm{N}}^{2}\left|x_{n_{\mathrm{t}}}-\hat{x}_{n_{\mathrm{t}}}\right|^{2}} .
$$




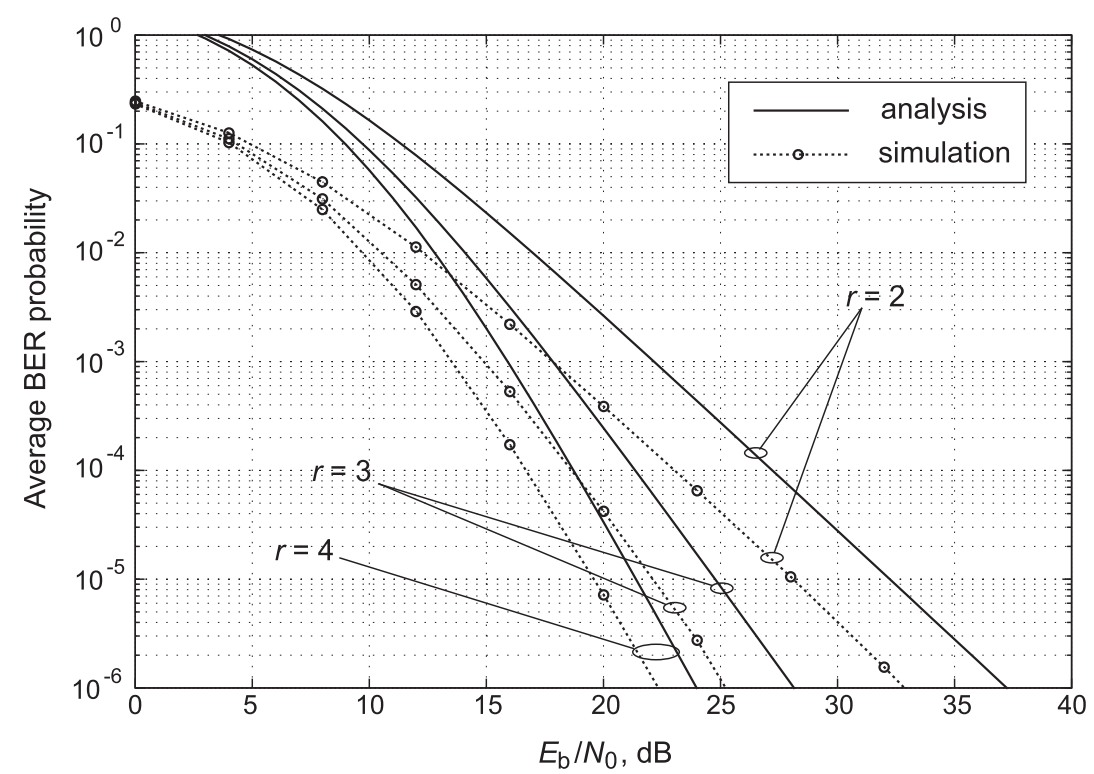

Fig. 1. Average BER probability versus $E_{\mathrm{b}} / N_{0}$ for differentially coherent detection in correlated Rayleigh fading channels.

Substituting Eq. (25) into Eq. (24), the bound is derived as

$$
P\left(x_{n_{\mathrm{t}}}, \hat{x}_{n_{\mathrm{t}}}\right) \leq \prod_{n_{\mathrm{R}}=1}^{N_{\mathrm{R}}} \frac{2 \sigma_{\mathrm{N}}^{2}\left(1+\frac{\left|m_{n_{\mathrm{R}}}\right|^{2}}{c_{n_{\mathrm{R}}}}\right) \exp \left(1-\frac{\left|m_{n_{\mathrm{R}}}\right|^{2}}{c_{n_{\mathrm{R}}}}\right)}{\left(c_{n_{\mathrm{R}}}+\left|m_{n_{\mathrm{R}}}\right|^{2}\right)\left|x_{n_{\mathrm{t}}}-\hat{x}_{n_{\mathrm{t}}}\right|^{2}} .
$$

The upper bound on the average BER probability is obtained by the union bound of the pairwise error probabilities as

$$
P\left(x_{n_{\mathrm{t}}}, \hat{x}_{n_{\mathrm{t}}}\right) \leq \sum_{x_{n_{\mathrm{t}}}, \hat{x}_{n_{\mathrm{t}}} \in \mathcal{X}} p\left(x_{n_{\mathrm{t}}}\right) N\left(x_{n_{\mathrm{t}}}, \hat{x}_{n_{\mathrm{t}}}\right) P\left(x_{n_{\mathrm{t}}}, \hat{x}_{n_{\mathrm{t}}}\right),
$$

where $p\left(x_{n_{\mathrm{t}}}\right)$ denotes the a priori probability of transmitting $x_{n_{\mathrm{t}}}$, and $N\left(x_{n_{\mathrm{t}}}, \hat{x}_{n_{\mathrm{t}}}\right)$ is the error ratio between $x_{n_{\mathrm{t}}}$ and $\hat{x}_{n_{\mathrm{t}}}$.

\section{Numerical example}

Numerical examples are presented to compare the analysis bounds with the actual BER probabilities. The average BER probability versus the bit energyto-noise ratio $E_{\mathrm{b}} / N_{0}$ from the analysis and simulation results in correlated Rayleigh fading channels is shown in Fig. 1 as a parameter of the matrix rank for the covariance of the fading channels given by $r=\operatorname{rank}(\boldsymbol{C})$, where $\operatorname{rank}(\cdot)$ denotes the matrix rank. The system differentially encodes the signal with quadrature phase-shift keying at the transmitter and receives the signals with $N_{\mathrm{R}}=4$ antennas. The Chernoff bound parameter optimized for the uncorrelated Rayleigh fading channel is applied to obtain the analysis bounds; hence, the results for ranks of $r=2$ and 3 are slightly worse than those in the use of the optimum bound parameter. To compute the precise bound requires to obtain the appropriate parameter by inspection. The difference between the results for a small number of diversity branches is mainly due to 


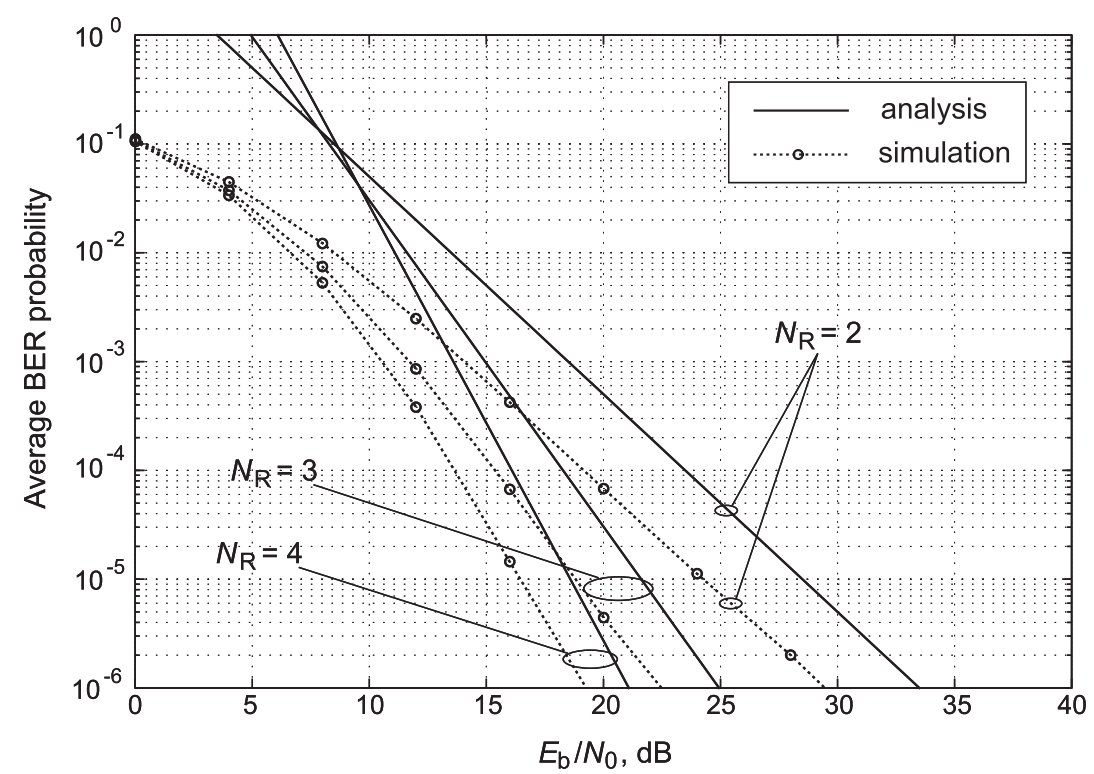

Fig. 2. Average BER probability versus $E_{\mathrm{b}} / N_{0}$ for coherent detection with channel phase information in uncorrelated Nakagami-Rice fading channels.

the looseness of the union bound. The results show that the analysis bounds approach to the actual values in the high $E_{\mathrm{b}} / N_{0}$ region and that the rank of the covariance matrix reflects the diversity gain. Since the correlated channel in this example has the relatively small spread of the eigenvalues, the effect of the matrix rank appears clearly.

The average BER probability versus $E_{\mathrm{b}} / N_{0}$ for coherent detection with channel phase information in uncorrelated Nakagami-Rice fading channels is shown in Fig. 2. All diversity links are assumed to have identical average fading power and $K$-factors of $K=0 \mathrm{~dB}$. The figure shows that the analysis results bound the actual values tightly in the high $E_{\mathrm{b}} / N_{0}$ region. The bound is seen to become tighter as increasing of the number of diversity links.

\section{Conclusion}

The reception diversity with coherent and differentially coherent detection has been studied. The upper bounds of the BER probabilities for differentially coherent detection in correlated Nakagami-Rice fading channels and for coherent detection with channel phase information in uncorrelated NakagamiRice fading channels were derived. The numerical examples verified the correctness of the analysis bounds. The results for differentially coherent detection confirmed that the rank of the covariance matrix of the channel reflects the diversity gain. 